\title{
Genetic testing in neurodevelopmental disorders
}

\author{
Jeremy Veenstra-VanderWeele, M.D. [Associate Professor of Psychiatry, Pediatrics, and \\ Pharmacology] \\ Vanderbilt Kennedy Center for Research on Human Development Vanderbilt Brain Institute 465 \\ $21^{\text {st }}$ Ave S, 7158 MRB III Nashville, TN 37212 615-936-1701 j.vvw@vanderbilt.edu
}

\section{Here: In This Issue}

\section{Volume 52, Number 5, May 2013}

This issue of the Journal draws our attention to the boundaries between different psychiatric disorders and potential biomarkers that may refine those boundaries.

Considerable controversy has surrounded the diagnosis of Bipolar Disorder (BD) in youth. Towbin and colleagues (p.XX) review the evidence for two distinct symptom patterns in children that share some similarities with BD but fall short of meeting DSM-IV criteria: Bipolar Disorder Not Otherwise Specified (BP-NOS) and Severe Mood Dysregulation (SMD). They examine BP-NOS as defined by the Course of Bipolar Youth (COBY) study, with most such children having manic, hypomanic, or mixed episodes shorter than the four days required for BD II. In contrast, they evaluate Severe Mood Dysregulation (SMD) as defined by the NIMH Intramural Research Program, which is distinguished by chronic irritability and hyperarousal symptoms without an episodic pattern of mood states. Notably, children with both BP-NOS and SMD show significant, chronic impairment that is comparable to $\mathrm{BD}$. The clearest distinction between the two is the episodic nature of BPNOS and the chronic pattern of SMD. First-degree relatives of youth with BP-NOS have a $25 \%$ rate of Bipolar Disorder, in contrast with a 3\% rate in first-degree relatives of SMD youth. Longitudinally, 25\% of children with BP-NOS meet criteria for Bipolar Disorder within 2 years (and $45 \%$ within 5 years); whereas $1.2 \%$ of youth with SMD cross the Bipolar Disorder threshold at 29 months. In sum, available research favors continuity between BP-NOS, as defined by COBY, and BD. Severe Mood Dysregulation, in contrast, co-occurs frequently with ADHD, ODD, and anxiety disorders but shows little connection with Bipolar Disorder. At this point, unfortunately, almost no research provides guidance on treatment of either BP-NOS or SMD.

Previous research in adult and childhood-onset schizophrenia (COS) has identified decreased hippocampal volume as a consistent biomarker, with more studies pointing to the anterior hippocampus than other sub-regions. Johnson and colleagues (p.XX) examine whether shape differences in the anterior hippocampus are seen in 103 individuals with COS and 79 healthy siblings of those individuals, in contrast with 101 healthy controls. They 
identified significant inward deformations in the anterior hippocampus in COS in contrast with healthy controls $(\mathrm{P}<0.05)$. Healthy siblings showed a similar pattern but only at a trend level after statistical correction. No significant difference was found between COS and healthy siblings, suggesting that this biomarker could correspond to a trait or endophenotype that may indicate familial susceptibility to COS, rather than an indicator of the disorder itself. Some relationships between hippocampal shape and symptoms were found, but none were statistically significant. Future work could examine whether similar shape changes are seen in other psychiatric disorders.

Psychiatric diagnoses often cluster together. In the Treatment of Resistant Depression in Adolescents (TORDIA) study, half of the participants had a comorbid diagnosis. Here, Hilton and colleagues (p.XX) assess improvements in comorbid anxiety disorder, attention deficit hyperactivity disorder (ADHD), or disruptive behavior disorder (DBD) symptoms in youth undergoing a medication switch, either with or without the addition of cognitive behavioral therapy (CBT). A previous TORDIA analysis found that the addition of CBT was particularly beneficial for youth with comorbid anxiety or ADHD symptoms. The present analysis turns this around to ask whether anxiety, ADHD, or DBD symptoms improve more with the addition of CBT, or whether they improve differentially with venlafaxine or a serotonin reuptake inhibitor. No significant advantage was seen for combined treatment or for any individual medication. Instead, remission of depression was the best predictor of improvement in comorbid anxiety disorder (remission by time interaction $\mathrm{P}=0.003$ ), ADHD ( $P=0.001)$, and DBD symptoms $(\mathrm{P}=0.048)$. Further work is needed to understand whether this indicates the generalized effect of successful treatment or, instead, the interference of persistent comorbid symptoms with depression treatment.

\section{There: Abstract Thinking}

\section{Genetic testing in neurodevelopmental disorders}

We are in the midst of a whirlwind of genetic research in neurodevelopmental disorders (NDs). Over the past decade, a variety of copy number variants (CNVs) - deletions or duplications ranging from thousands to millions of nucleotides - have been implicated in NDs. The American College of Medical Genetics (ACMG) now recommends that every person with developmental delay, Intellectual Disability, or Autism Spectrum Disorder (ASD) should receive a Chromosomal Microarray (CMA) as the first-line test to identify CNVs that contribute to risk in $10-15 \%$ of individuals ${ }^{1}$.

Another source of risk for NDs has been revealed by next generation DNA sequencing that allows every exon of every gene (the "whole exome") to be examined simultaneously. Each of our exomes contains many single nucleotide variants (SNVs) that disrupt a single copy of a gene, but Lim and colleagues ${ }^{2}$ found that children with ASD were twice as likely ( $\mathrm{P}=$ 0.0017) to have SNVs disrupting both copies of a gene, suggesting recessive inheritance of ASD risk. Similarly, they found that males with ASD were more likely than controls to have a disruptive $\mathrm{SNV}$ on the $\mathrm{X}$ chromosome (OR 1.5, $\mathrm{P}=0.034)$. Overall, the affected genes were quite diverse, but the odds ratio (OR) was higher for genes expressed in brain (OR 2.7, $\mathrm{P}=0.002)$ than for those not expressed in brain ( $\mathrm{OR} 1.5, \mathrm{P}=0.29)$. From a clinical perspective, this sequencing effort also yielded molecular diagnosis of previously undetected 
syndromes in some participants. The authors estimate the risk attributable to these recessively inherited variants as accounting for $5 \%$ of ASD. Combined with the risk attributed to CNVs as well as the $10 \%$ risk attributed to de novo SNVs (non-inherited mutations), these rare variants collectively are pretty common in ASD.

For clinicians, the questions around genetic testing center on utility. Clearly, having a molecular diagnosis is useful for family planning. But will a molecular diagnosis change treatment for the child with an ND? Will it do so in the present tense, or in the distant future? A few recent studies show that molecular diagnosis may yield new treatment options. For example, in three families segregating ASD, ID, and epilepsy, Novarino and colleagues $^{3}$ identified recessively inherited SNVs in a gene, $B C K D K$, that regulates metabolism of branched-chain amino acids (BCAAs). Children with disruption of this gene on both chromosomes showed decreased BCAAs in their blood. Paralleling this finding, mice lacking this gene showed decreased BCAAs in the brain, resulting in epilepsy, limb clasping, and impaired growth. In mice, all of these abnormalities were rescued by supplementation with BCAAs, suggesting this as a potentially treatable disorder identified by exome sequencing. Just as the CMA replaced the karyotype a few years ago, whole exome or genome sequencing is likely to become the first-line test in NDs within the next few years.

\section{Acknowledgments}

I thank Dr. Natalie Weder for helpful editorial suggestions. This work was supported in part by NIH grant R01MH094604.

\section{References}

1. Miller DT, Adam MP, Aradhya S, et al. Consensus statement: chromosomal microarray is a first-tier clinical diagnostic test for individuals with developmental disabilities or congenital anomalies. Am J Hum Genet. 2010; 86(5):749-764. [PubMed: 20466091]

2. Lim ET, Raychaudhuri S, Sanders SJ, et al. Rare complete knockouts in humans: population distribution and significant role in autism spectrum disorders. Neuron. 2013; 77(2):235-242. [PubMed: 23352160]

3. Novarino G, El-Fishawy P, Kayserili H, et al. Mutations in BCKD-kinase lead to a potentially treatable form of autism with epilepsy. Science. 2012; 338(6105):394-397. [PubMed: 22956686] 